\title{
LA EMISIÓN FOLIAR EN PLÁTANO Y SU RELACIÓN CON LA DIFERENCIACIÓN FLORAL ${ }^{1}$
}

\author{
Alfonso Vargas-Calvo ${ }^{2}$, Pablo Acuña-Chinchilla ${ }^{3}$,Henry Valle-Ruiz ${ }^{4}$
}

\begin{abstract}
RESUMEN
La emisión foliar en plátano y su relación con la diferenciación floral. El objetivo del presente trabajo fue estudiar la emisión foliar de plátanos del tipo Falso Cuerno y su relación con el proceso de la diferenciación floral. Se efectuaron cuatro estudios de noviembre del 2000 a enero del 2014, bajo las condiciones de la región Caribe de Costa Rica. Se emplearon registros del tipo y número de hojas emitidas. El cambio del meristemo se determinó por la disección de las plantas a diferentes etapas de emisión foliar. En el Estudio I (cvs. Hartón alto y Hartón enano, segundo ciclo productivo) la emisión foliar fue similar entre cultivares altos $(P>0,2417)$, enanos $(P>0,05735)$ y entre ambos $(\mathrm{P}>0,4170)$. En el Estudio II (cv. Hartón alto, primer ciclo productivo) el meristemo en fase vegetativa se observó en el 100\% de las plantas hasta la emisión de la hoja 21 inclusive. El mayor porcentaje de plantas en fase de transición $(55,60,72$ y $60 \%)$ ocurrió a las 24, 25, 26 y 27 hojas verdaderas. En el Estudio III (cvs. Hartón alto y Hartón enano, tercer ciclo sucesorio productivo) la diferenciación se observó a partir de la hoja 20 en el 10\% y en el $60 \%$ de las plantas de los cultivares de porte alto y bajo, respectivamente. En la hoja 24 el 100\% de las plantas disectadas estaba en transición o en diferentes etapas de la fase reproductiva. En el Estudio IV (cv. Hartón alto, tercer ciclo sucesorio productivo) no fue posible relacionar la fase de diferenciación floral con el cambio morfológico en la base de los semilimbos de la hoja.
\end{abstract}

Palabras clave: diferenciación celular, morfología de la planta, Musa (plátanos), meristemas apicales.

\begin{abstract}
Plantain foliar emission and its relationship with floral differentiation. The objective of this study was to determine the relationship between foliar emission and floral differentiation in False Horn plantains. Four studies were carried out on the Caribbean coast of Costa Rica between November 2000 and January 2014. Recoded variables were type and number of emitted leaves. In Study I (tall and dwarf cultivars, first ratoon) leaf emission was similar among tall $(\mathrm{P}=0.2417)$ and dwarf $(\mathrm{P}=0.5735)$ cultivars and between both of them ( $\mathrm{P}=0.4170)$. In Study II (cv. Harton tall, plant crop) the vegetative phase of the meristem was observed in $100 \%$ of plants until the emission of leaf 21 . The highest percentages of plants in which the meristem was in transition stages was $55 \%, 60 \%, 72 \%$ and $60 \%$, and occurred in plants with 24, 25, 26 and 27 leaves, respectively. In Study III (cvs. Hartón tall and Hartón dwarf, second ratoom) differentiation was observed from leaf 20 in 10\% and 60\% of plants of tall and dwarf cultivars, respectively. A 100\% of the dissected plants were in transition or at different stages of the reproductive phase by the time they reached leaf 24 . In Study IV (Harton tall, second ratoon) it was not possible to find a relationship between the floral differentiation phase and the morphological change in the basal blades of the leaf.
\end{abstract}

Keywords: cell differentiation, plant anatomy, Musa (plantains), apical meristems.

\footnotetext{
Recibido: 22 de julio, 2014. Aceptado: 9 de setiembre, 2014. Proyecto de investigación. Corporación Bananera Nacional.

2 Corporación Bananera Nacional (CORBANA, S.A). Apdo. 390-7210, Guápiles, Costa Rica. alfvarga@ corbana.co.cr

3 Instituto Nacional de Innovación y Transferencia Agropecuaria (INTA). Apdo. 382-1000, San José, Costa Rica. pacuna@inta.go.cr

4 Abonos del Pacífico, Costa Rica. Apdo. 10929-1000, San José,Costa Rica. henry.valle@abopac.com
} 


\section{INTRODUCCIÓN}

El número de hojas que puede emitir la planta de plátano (cv. Dominico Hartón) durante su ciclo vegetativo es de $38 \pm 2$ y dicha cantidad es inversamente proporcional al tamaño y peso del cormo donde, a un menor peso de cormo mayor número de hojas y viceversa (Belalcázar et al., 1991). Esta emisión foliar de acuerdo con Belalcázar y Valencia (1998) es concordante con diferentes cultivares del tipo Falso Cuerno. En ambos casos, independientemente del tamaño del material de siembra o de su origen, las plantas de primer ciclo de producción (generadas a partir del crecimiento del material sembrado), emitirán sólo hojas verdaderas (con 10 o más $\mathrm{cm}$ en su parte más ancha); mientras que en los siguientes ciclos sucesorios de producción, se formarán hojas filiformes (de 1 a menos de $10 \mathrm{~cm}$ en su parte más ancha) y hojas verdaderas. Esta particularidad se debe considerar de manera prioritaria para una adecuada interpretación de los datos generados en los estudios sobre la fase de transición del meristemo de vegetativo a reproductivo.

La diferenciación floral inicia cuando el meristemo vegetativo se transforma en reproductivo, con el cese de la emisión de hojas y el comienzo de la formación de las partes florales (Turner et al., 2007), fase denominada también como de transición. Los primeros estudios sobre diferenciación floral, todos efectuados en cultivares de banano de los subgrupos Gros Michel y Cavendish, fueron desarrollados por Alexandrowicz (1955) quien describió los primeros cambios morfológicos del meristemo, y por Summerville (1944) quien relacionó el proceso de la diferenciación floral con la producción y longevidad del área foliar, la temperatura y la duración de la luz solar. En este último aspecto, Turner et al. (2007) señalan que aunque la planta de banano es neutra (no depende del fotoperiodo para la inducción floral), fotoperiodos de menos de doce horas están asociados, independientemente de la temperatura, con una demora en la tasa de diferenciación floral.

La asociación de la emisión foliar con la fase de transición ha sido determinada en banano por diversos autores entre las hojas veinte a veinticinco con seis a once de ellas aun por emerger del pseudotallo (Dumas, 1955), con la emisión de más de diceisiete hojas con once a doce hojas por emerger del pseudotallo (Ganry, 1973), entre veintitrés a veinticuatro hojas (veintinueve hojas en promedio) con seis a diecisiés hojas (11,9 hojas en promedio) presentes aun sin emerger dentro del pseudotallo (Stover, 1979), entre 32 y 33 hojas para los cvs Grande Naine, Williams y Valery en plantas de primer ciclo de producción de cultivo in vitro (Valle et al. 2007) y entre diecisiete a diecinueve hojas verdaderas con doce hojas aún por emerger del pseudotallo en plantas de cuarto ciclo sucesorio de producción de los tres cultivares antes citados (Valle y González, 2009).

En plátanos del tipo Falso Cuerno (cv. Dominico Hartón) cultivados entre 1300 a 1600 msnm, Belalcázar et al. (1991) mencionan que la transición floral sucede alrededor de los cinco o seis meses de iniciado el proceso de producción de hojas con la emisión del 50\% del total de las mismas (alrededor de las diecinueve hojas). Mientras que Aristizábal y Jaramillo (2010) indican que para el mismo cultivar sembrado a 1050 $\mathrm{msnm}$, dicha etapa ocurre cuando la planta ha emitido veintisiete hojas. Por su parte, Hernández et al. (2010) en Venezuela, ubica la transición floral en plátanos (cv. Hartón enano) del mismo tipo (Falso Cuerno) en la hoja 25 (33\% de las plantas), en la hoja 27 (60\% de las plantas) y en la hoja 30 (80-100\% de las plantas). Estos últimos autores mencionan que al originarse la diferenciación floral se inicia el crecimiento del tallo verdadero en cuyo ápice se encuentra la inflorescencia, la cual se traslada por el centro del pseudotallo hasta emerger por la parte superior de la planta.

La diferenciación floral en plátano guarda relación con un determinado número de hojas emitidas y está relacionado con los cambios morfológicos de la hoja (Belalcázar et al., 1991). Así, de acuerdo con el mismo autor, aproximadamente hasta la hoja 17, la inserción de los semilimbos con la nervadura central es simétrica y en consecuencia el ángulo es muy cerrado. Sin embargo, con cada una de las hojas emitidas posteriormente, el ángulo se va abriendo y la inserción de los dos semilimbos es cada vez más asimétrica, situación que es mucho más visible y marcada en la hoja 22. No obstante, Hernández et al. (2010) mencionan que aún no se ha logrado establecer en el campo, de manera visual, cuándo ocurre la transición floral, debido a que la planta no manifiesta algún índice notable en su exterior. Sin embargo, la identificación de la etapa en que dicho proceso podría estimarse con precisión, mediante el estudio de la relación entre el número de hojas verdaderas emitidas 
y la ocurrencia del proceso en el interior de la planta. La consideración de este aspecto en virtud de los nuevos sistemas de producción intensivos de plátano recomendados (Belalcázar et al., 1991; Vargas, 1994 y 1995) y, basados en la alta densidad de población y un ciclo productivo, proveería una herramienta para lograr un manejo agronómico más eficiente y racional.

El objetivo del presente trabajo fue estudiar la emisión foliar en plátanos del tipo Falso Cuerno y su relación con el proceso de la diferenciación floral.

\section{MATERIALES Y MÉTODOS}

Se efectuaron cuatro estudios con plátanos del tipo Falso Cuerno (Musa AAB) en el Caribe de Costa Rica. Los estudios I, III y IV se localizaron en el Centro de Investigación Agrícola 28 Millas (10 5' 52 " latitud Norte y $83^{\circ} 22^{\prime} 33^{\prime}$ ' longitud Oeste, 27 msnm) y se efectuaron en diferentes momentos sobre un mismo terreno conformado en domos; mientras que el estudio II se ubicó en el Centro de Investigación Agrícola La Rita (10 16' 28' latitud Norte y 83 46' 32" longitud Oeste, $106 \mathrm{msnm}$ ). El suelo de 28 Millas es de origen sedimentario, de alta fertilidad natural, $\mathrm{pH}$ moderadamente ácido, bajos porcentajes de materia orgánica, altos contenidos de $\mathrm{Ca}$ y $\mathrm{Mg}$ con respecto al K. El suelo de La Rita es, de origen volcánico, de baja fertilidad natural, $\mathrm{pH}$ ácido, altos porcentajes de materia orgánica, bajos contenidos de $\mathrm{Ca}$ y $\mathrm{Mg}$ con respecto a los de K (López y Solís, 1991).

La fertilización en 28 Millas (zona Este) se realizó considerando la emisión foliar con siete aplicaciones por planta, donde las tres primeras se efectuaron cuando estas emitieron de cuatro a nueve hojas con $18 \mathrm{~g}$ de sulfato de amonio $\left(21 \% \mathrm{~N} ; 23,7 \% \mathrm{SO}_{4}\right)$, las dos siguientes a la emisión de diez a trece hojas con $23 \mathrm{~g}$ de 15-3-31 ( $\left.-\mathrm{P}_{2} \mathrm{O}_{5}-\mathrm{K}_{2} \mathrm{O}\right)$ y las dos restantes a la emisión de quince a veinticinco hojas con $30 \mathrm{~g}$ de $15-$ 3-31. En La Rita (zona oeste) la fertilización se realizó mensualmente con $25 \mathrm{~g} /$ planta de sulfato de amonio $\left(21 \% \mathrm{~N} ; 23,7 \% \mathrm{SO}_{4}\right)$ y $15 \mathrm{~g}$ de $\mathrm{KCl}\left(50 \% \mathrm{~K}_{2} \mathrm{O} ; 47 \%\right.$ $\mathrm{Cl}$ ). Además se aplicó $200 \mathrm{~g} / \mathrm{planta}$ de $\mathrm{CaCO}_{3}$ (39 a $40 \% \mathrm{Ca}$ ) cinco meses después de la siembra.

Según el Instituto Meteorológico Nacional (2008), el clima de la región Caribe no presenta una estación seca definida, pues las lluvias se mantienen entre los 100 y 200 mm en los meses menos lluviosos, condición que implica una alta cantidad de lluvia anual. De manera general Vargas y Blanco (2004) y Serrano et al. (2008) definen cuatro períodos climáticos durante el año: 1- lluvioso y frío (noviembre, diciembre y enero) con temperatura promedio de $24{ }^{\circ} \mathrm{C}$ y precipitación acumulada de $830 \mathrm{~mm}, 2$ - seco y caliente (febrero, marzo y abril) con temperatura promedio de $25,1{ }^{\circ} \mathrm{C}$ y precipitación acumulada de $609 \mathrm{~mm}$, 3- lluvioso y caliente (mayo, junio y julio) con temperatura promedio de $26,0{ }^{\circ} \mathrm{C}$ y precipitación acumulada de $1029 \mathrm{~mm}$ y 4- seco y fresco (agosto, setiembre y octubre) con temperatura promedio de $25,8{ }^{\circ} \mathrm{C}$ y precipitación acumulada de $690 \mathrm{~mm}$. Los dos primeros períodos conforman la época climática adversa y los dos últimos la favorable para el crecimiento de la planta y el desarrollo del racimo.

El combate de la Sigatoka negra (Mycosphaerella fijiensis) se realizó con aplicaciones rotativas de fungicidas protectores y sistémicos en mezcla (emulsión) con aceite agrícola (3,0 a 7,0 1/ha) y agua para un volumen total de aplicación entre 18,0 a 24,0 1/ha aplicado con una motobomba nebulizadora terrestre. El combate químico se complementó con la recolección del material vegetal proveniente de la deshoja sanitaria y el posterior traslado lejos del área de cultivo. El combate de malezas se realizó por medio de chapeas con machete o motoguadaña.

El combate químico de nematodos y picudos no se realizó en el estudio I mientras que en el II, III y IV se efectuó mediante la aplicación de terbufos (3 g i.a.); además, se realizaron trampas de cuña del mismo pseudotallo para la captura de los picudos.

La disección de plantas (estudios II, III y IV) consideró la eliminación total del dosel y la parcial del pseudotallo, el cual se cortó a una altura de 10 cm sobre el suelo. La porción de cormo y pseudotallo extraída se disectó y redujo hasta obtener un cilindro interno que contenía el meristemo apical. Estas fueron almacenadas en bolsas plásticas transparentes de 80 $\mathrm{cm}$ de altura y $60 \mathrm{~cm}$ de ancho, luego se transportaron, en contenedores con hielo para evitar la desecación del tejido, al Laboratorio de Biotecnología de CORBANA S.A. en La Rita. Posteriormente la muestra fue lavada con abundante agua y se redujo a un tamaño de entre $3,0 \times 3,0 \mathrm{~cm}$ hasta $6,0 \times 6,0 \mathrm{~cm}$ hasta obtener el tejido circundante. Luego se sumergió en una solución antioxidante de ácido ascórbico (10 g/l) y agua destilada para reducir la oxidación del tejido 
expuesto al corte. Los cortes obtenidos se observaron en el estereoscopio y las fotografías de aquellos seleccionados se tomaron con una cámara digital.

A continuación se describen las características específicas seguidas en cada uno de los cuatro estudios.

Estudio I- Determinación de la emisión foliar. Se realizó de noviembre del 2000 a marzo del 2001 en un área experimental de plantas (1600 plantas/ha) de segundo ciclo sucesorio de producción. Se evaluaron los cultivares de porte alto Dominico Hartón, Hartón, Tallo Rojo y Tallo Verde, así como las introducciones locales de porte alto: Cóbano, Joel, Pérez y Taylor también se evaluaron los cultivares de porte bajo Enano y Planta Baja II, cuya procedencia en particular fue descrita por Vargas et al. (2005). El diseño experimental fue de bloques completos al azar con los cultivares como tratamientos y cuatro repeticiones de cuatro plantas útiles cada una. Las variables medidas fueron: número de hojas filiformes (de 1 a menos de 10 $\mathrm{cm}$ en su parte más ancha) y de hojas verdaderas (más de $10 \mathrm{~cm}$ en su parte más ancha). La suma de ambas constituyó el número de hojas emitidas. Los datos se analizaron mediante ANDEVA y contrastes con el programa estadístico SAS (SAS Institute, 2004).

Estudio II- Relación entre la emisión foliar y la diferenciación floral (plantas de primera generación). Se efectuó de noviembre del 2009 a mayo del 2010 en un área de plantas (2500 plantas/ ha) de primer ciclo de producción del cv. Hartón alto. La sustracción de plantas se realizó en intervalos de una hoja a partir de la 20 y hasta la 35 con una cantidad disectada de 13 a 39 plantas por grupo, de acuerdo con la emisión foliar respectiva. En cada uno de los grupos se identificaron las fases vegetativas, de transición, reproductiva y de floración, de acuerdo con su ocurrencia, para un total de 406 plantas. Los datos de cada estado fenológico y de emisión foliar se relacionaron mediante un análisis descriptivo que consideró promedios y distribuciones de frecuencia.

Estudio III- Relación entre la emisión foliar y la diferenciación floral (plantas de tercera generación). El estudio se efectuó de mayo del 2013 a enero del 2014 en un área de plantas (2500 plantas/ha) de tercer ciclo sucesorio de producción de los cvs. Hartón alto y Hartón enano. La sustracción de plantas se efectuó en intervalos de dos hojas a partir de la hoja verdadera 22 y hasta la hoja verdadera 28 en cultivares de porte alto y hasta la hoja verdadera 26 en los porte bajo, con una cantidad de plantas por grupo de 11 a 14 en el primer caso y de 10 a 16 en el segundo. Conforme cada planta alcanzó el número requerido de hojas verdaderas emitidas, se identificó la fase fenológica (vegetativa o reproductiva) y se midió la longitud del meristemo diferenciado. Los datos de las etapas fenológicas y su emisión foliar se relacionaron mediante un análisis descriptivo que consideró promedios máximos y mínimos y distribuciones de frecuencia.

Estudio IV- Relación de los cambios morfológicos de la hoja con la diferenciación floral. Se desarrolló de noviembre del 2012 a julio del 2013 en un área de plantas (2500 plantas/ha) generadas a partir de plantas de tercer ciclo sucesorio de producción del cv Hartón alto. En cada planta se registró el número de hoja emitida en la cual se inició el cambio morfológico del primer semilimbo, así como aquel donde finalizó (de aguzado a redondeado) en el segundo semilimbo. En este último estado, se procedió a la extracción de plantas y a la determinación de la fase fenológica presente (vegetativa o reproductiva). Los datos de las etapas fenológicas con relación al inicio y al final del cambio morfológico de los limbos, se relacionaron mediante un análisis descriptivo que consideró promedios y distribuciones de frecuencia.

\section{RESULTADOS Y DISCUSIÓN}

Estudio I- Determinación de la emisión foliar. El número de hojas filiformes fue similar entre los cultivares de porte alto $(10,1$ a 11,0 hojas; $\mathrm{P}=0,5552)$ y entre los de porte bajo (10,7 a 10,9 hojas; $\mathrm{P}=0,7101)$. El número de hojas verdaderas no varió en los cultivares de porte alto $(27,6$ a 28,9 hojas; $P=0,5193)$ ni en los de porte bajo (28,0 a 28,5 hojas; $\mathrm{P}=0,5735)$. La cantidad de hojas totales emitidas (filiformes y verdaderas) fue similar entre los cultivares de porte alto $(38,0$ a 39,8 hojas; $\mathrm{P}=0,2417)$ y los de porte bajo (38,9 a 39,2 hojas; $\mathrm{P}=0,8836)$. El número de hojas filiformes $(\mathrm{P}=0,4170)$, verdaderas $(\mathrm{P}=0,8161)$ y totales $(\mathrm{P}=0,7748)$ no varió entre ambos portes de planta (Cuadro 1).

Los diferentes cultivares del tipo Falso Cuerno tanto de porte alto como de porte bajo $(27 \mathrm{msnm})$ presentaron similar número de hojas emitidas, coincide con lo señalado por Belalcázar et al. (1991) para una zona cafetalera de Colombia $(1320 \mathrm{msnm})$ con el cultivar, también del tipo Falso Cuerno, denominado Dominico Hartón. No obstante, en el primer caso, dado 
Cuadro 1. Emisión foliar de nueve cultivares de plátano del tipo Falso Cuerno (Musa AAB) de porte alto y de porte bajo. Estudio I. Matina, Limón, Costa Rica. 2000-2001.

\begin{tabular}{|c|c|c|c|}
\hline Cultivar & $\begin{array}{c}\text { Hojas } \\
\text { filiformes }\end{array}$ & $\begin{array}{c}\text { Hojas } \\
\text { verdaderas }\end{array}$ & $\begin{array}{l}\text { Hojas } \\
\text { totales }\end{array}$ \\
\hline \multicolumn{4}{|l|}{ Porte alto } \\
\hline Cóbano & 10,6 & 28,4 & 39,0 \\
\hline Dominico & 10,1 & 28,2 & 38,2 \\
\hline \multicolumn{4}{|l|}{ Hartón } \\
\hline Joel & 10,7 & 28,9 & 39,6 \\
\hline Pérez & 10,4 & 27,6 & 38,0 \\
\hline Tallo Rojo & 11,0 & 28,8 & 39,8 \\
\hline Tallo Verde & 10,1 & 28,1 & 38,2 \\
\hline Taylor & 10,8 & 28,5 & 39,3 \\
\hline \multicolumn{4}{|l|}{ Porte bajo } \\
\hline Enano & 10,7 & 28,5 & 39,2 \\
\hline Planta Baja II & 10,9 & 28,0 & 38,9 \\
\hline \multicolumn{4}{|c|}{ Contrastes (probabilidades) } \\
\hline Entre porte alto & 0,5552 & 0,5193 & 0,2417 \\
\hline Entre porte bajo & 0,7101 & 0,5735 & 0,8836 \\
\hline $\begin{array}{l}\text { Porte alto } v s \\
\text { porte bajo }\end{array}$ & 0,4170 & 0,8161 & 0,7748 \\
\hline
\end{tabular}

que las plantas medidas correspondieron a una sucesión (segundo ciclo de producción), la emisión foliar estuvo conformada por hojas filiformes y verdaderas, mientras que en el segundo caso, estuvo constituida por plantas de primera generación, con una emisión total de solo hojas verdaderas. Esto indica que aunque la emisión foliar total en ambos casos fue similar, no ocurrió lo mismo con respecto a la cantidad de hojas verdaderas, aspecto que pone de manifiesto, con el fin de evitar confusiones, la necesidad de relacionar la transición del meristemo con este tipo de hoja, cuya percepción es más práctica y sencilla en el campo.

Asimismo, la comparación de los resultados obtenidos en este estudio y los señalados por Belalcázar et al. (1991), permiten inferir que la cantidad total de hojas producidas por la planta, en contraste con su tasa de emisión, no sería afectada por las condiciones agroclimáticas dadas en virtud de la altitud.
Estudio II- Relación entre la emisión foliar y la diferenciación floral (plantas de primera generación). En las plantas disectadas (Cuadro 2) el meristemo en fase vegetativa se observó en el $100 \%$ de las plantas hasta la hoja 21 inclusive. A partir de esta hoja en cada grupo de plantas hubo una reducción de este porcentaje y un aumento paulatino en el porcentaje de plantas en las que se detectó la fase de transición. El porcentaje predominante de plantas cuyo meristemo estuvo en fase vegetativa al momento de la disección (85 y $60 \%$ ) ocurrió en las hojas 22 y 23, respectivamente; mientras que el mayor porcentaje de plantas cuyo meristemo estuvo en la fase de transición $(55,60,72$ y $60 \%)$ ocurrió en las plantas disectadas a las $24,25,26$ y 27 hojas, respectivamente.

Ocurrido el cambio del meristemo vegetativo a reproductivo, el desarrollo de la inflorescencia dentro del pseudotallo, se observó en la mayoría de las plantas disectadas a las 28,29 y 30 hojas $(72,90$ y $95 \%$, del total de plantas disectadas, respectivamente). A partir de la emisión de la hoja 30 y hasta la hoja 33, la proporción de plantas con desarrollo de inflorescencia decreció con cada hoja emitida (de 95 a 55\%). En las hojas 34 y 35 también aparecieron las últimas inflorescencias en desarrollo, pero ya en una proporción menor (36 y $18 \%$, respectivamente) del total del grupo de plantas extraídas. La emergencia de la inflorescencia se inició a partir de la emisión de la hoja 30 (5\%), pero el porcentaje predominante se determinó a la emisión de las hojas 34,35 y 36 (64, 82 y $100 \%$, respectivamente).

La observación en el estereoscopio de los cortes, mostró al meristemo apical vegetativo ubicado en posición central superior del cormo, protegido por los primordios foliares envolventes en arreglo helicoidal (Figura 1A). El meristemo vegetativo estuvo por debajo o al mismo nivel que la base de los primordios foliares más próximos mientras que en la fase de transición comenzó a subir y tomar una posición superior a los primordios. En las plantas disectadas entre las hojas 24 a 27 se observó una elevación del punto central formando un cono o triángulo (Figura 1B). En la fase reproductiva (hojas 28 y 29), el ápice floral ocupó una posición superior central sobre un cilindro de tejido de reserva del cormo que se proyectó acrópetamente, y el meristemo floral derivó en forma helicoidal las estructuras de la inflorescencia, caracterizadas por primordios de brácteas florales con flores en las axilas (Figura 1C). 
Cuadro 2. Etapas fenológicas antes, durante y después de la fase de transición del meristemo en grupos de plantas de plátano del tipo Falso Cuerno (Musa AAB, cv. Hartón alto). Estudio II. Pococí, Limón, Costa Rica. 2009-2010.

\begin{tabular}{|c|c|c|c|c|c|c|c|c|c|}
\hline \multirow[b]{2}{*}{$\mathbf{H E}^{1}$} & \multirow[b]{2}{*}{$\underset{\text { (total) }}{\mathbf{n}}$} & \multicolumn{8}{|c|}{ Proporción de plantas en cada etapa fenológica (\%) } \\
\hline & & $\begin{array}{l}\text { Vegetativa } \\
\text { (\% de } \\
\text { plantas) }\end{array}$ & nef & $\begin{array}{c}\text { Transición } \\
\text { (\% de } \\
\text { plantas) }\end{array}$ & nef & $\begin{array}{c}\text { Reproductiva } \\
(\% \text { de } \\
\text { plantas })\end{array}$ & nef & $\begin{array}{c}\text { Floración } \\
\text { (\% de } \\
\text { plantas) }\end{array}$ & nef \\
\hline 20 & 20 & 100 & 20 & & & & & & \\
\hline 21 & 13 & 100 & 13 & & & & & & \\
\hline 22 & 20 & 85 & 17 & 15 & 3 & & & & \\
\hline 23 & 10 & 60 & 6 & 40 & 4 & & & & \\
\hline 24 & 20 & 40 & 8 & 55 & 11 & 5 & 1 & & \\
\hline 25 & 20 & 35 & 7 & 60 & 12 & 5 & 1 & & \\
\hline 26 & 25 & 16 & 4 & 72 & 18 & 12 & 3 & & \\
\hline 27 & 20 & 10 & 2 & 60 & 12 & 30 & 6 & & \\
\hline 28 & 25 & 4 & 1 & 24 & 6 & 72 & 18 & & \\
\hline 29 & 20 & & & 10 & 2 & 90 & 18 & & \\
\hline 30 & 20 & & & & & 95 & 19 & 5 & 1 \\
\hline 31 & 25 & & & & & 92 & 23 & 8 & 2 \\
\hline 32 & 20 & & & & & 85 & 17 & 15 & 3 \\
\hline 33 & 20 & & & & & 55 & 11 & 45 & 9 \\
\hline 34 & 36 & & & & & 36 & 13 & 64 & 23 \\
\hline 35 & 39 & & & & & 18 & 7 & 82 & 32 \\
\hline 36 & 53 & & & & & & & 100 & 53 \\
\hline
\end{tabular}

${ }^{1}$ HE: hojas emitidas (emisión foliar) al momento de la disección. n: número total de plantas disectadas para cada emisión foliar. nef: número individual de plantas para cada etapa fenológica.

En el cultivo intensivo del plátano la obtención de rendimientos superiores se basa en el uso de altas densidades de población en conjunto con la renovación total de la plantación luego de cada ciclo de cultivo (Belalcázar et al., 1991; Vargas 1994, 1995). Este concepto demanda una alta uniformidad de la plantación, condición ideal para programar y racionalizar, con base en la ocurrencia del proceso de transición del meristemo, el manejo agronómico de estas plantas de primera generación, cuya emisión foliar, está compuesta totalmente de hojas verdaderas. En estas plantas la transición foliar ha sido señalada entre las hojas 20 a 25 (Dumas, 1995) y 23 a 24 (Stover, 1979) en banano y de 25 a 30 (Hernández et al., 2010) en plátano Hartón enano, valores que incluyen el rango identificado (veinticuatro a veintisiete hojas verdaderas) en el cv. de plátano usado (Hartón alto) en el presente estudio.

Existen productores de plátano que utilizan al menos un ciclo de producción sucesorio más, cuyo hijo de sucesión emite una serie de láminas foliares de morfología diferente, que va desde filiformes a verdaderas; considerando verdaderas aquellas con más de $10 \mathrm{~cm}$ en la parte más ancha. Dado que la emisión foliar se asocia por lo general con un número de hojas emitidas, generalmente verdaderas, la consideración de este intervalo en plantas sucesoras debería estar siempre en función de dichas hojas. Situación similar ocurre en plantas de banano originadas de cormos (Ganry, 1973) o provenientes de cultivo in vitro (Valle y González, 2009), así como de plantas de plátano obtenidas de cormos (Belalcázar et al., 1991), 

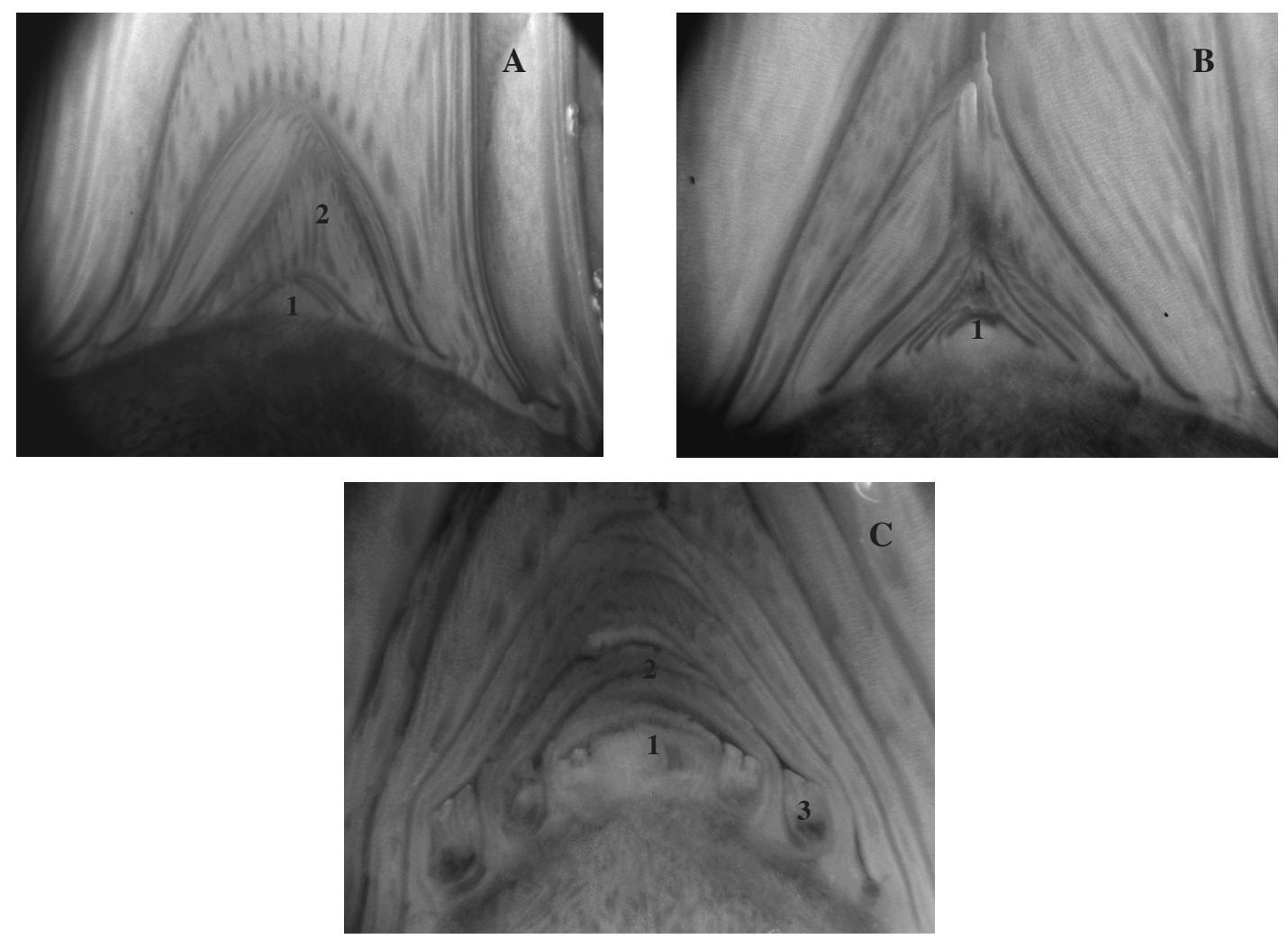

Figura 1. Zona apical de plantas de plátano (Musa AAB) del subgrupo Falso Cuerno: A- meristemo apical vegetativo (1) cubierto por los primordios foliares (2). B- meristemo apical en fase de transición (1) mostrando una posición ascendente. C- meristemo apical reproductivo (1) con primordios de brácteas florales laterales (2) y primordios de frutos (3) en las axilas. Estudio II. Pococí, Limón, Costa Rica. 2009- 2010.

en las cuales el número de hojas verdaderas para ambos genomas (AAA y $\mathrm{AAB}$ ) varió entre diecisiete y diecinueve hojas.

Estudio III- Relación entre la emisión foliar y la diferenciación floral (plantas de tercera generación). En las plantas disectadas (Cuadro 3) a las veinte hojas verdaderas el meristemo se observó en la fase de transición en un $10 \%$ de las plantas del cv. de porte alto y en un $60 \%$ de aquellas de porte bajo, con un $100 \%$ en ambos casos, y con diferente magnitud de la fase reproductiva a partir de la hoja 24 . La altura desde la sección superior del cormo donde se origina el ápice hasta la parte distal, ya diferenciada del mismo, estuvo comprendida para la hoja 20 en las diferentes plantas del cv. de porte alto entre 0,2 (valor mínimo observado) y $0,6 \mathrm{~cm}$ (valor máximo observado). Estos valores fueron aumentando con cada grupo, especialmente para las disecciones en las plantas con veintiséis hojas $(21$ y $283 \mathrm{~cm})$ y veintiocho hojas $(12$ y $258 \mathrm{~cm})$. Un comportamiento similar se determinó en la hoja 20 del cv. de porte bajo, ya que los valores mínimos y máximos fueron de 0,2 y $1,1 \mathrm{~cm}$ respectivamente; y en la hoja 26 de 2,5 y $150 \mathrm{~cm}$ (Cuadro 3).

El inicio de la fase de transición en la hoja verdadera 20, con un mayor porcentaje de ocurrencia en el cultivar de porte bajo, se podría relacionar con la duración del ciclo de vida que muestran los diferentes cultivares de plátano. Al respecto, Vargas y Guzmán (2001) indican mayor precocidad del cultivar de porte bajo.

Considerando la cantidad de hojas verdaderas promedio a la floración que se determinaron en el estudio I, tanto para los cultivares de porte alto como para los de porte bajo (28,3 hojas), en las plantas disectadas a veinte hojas restarían 8,3 hojas para la 
Cuadro 3. Relación entre la emisión foliar de hojas verdaderas y la diferenciación floral en plantas de plátano (Musa AAB) del tipo Falso Cuerno (cvs. Hartón alto y Hartón enano). Estudio III. Matina, Limón, Costa Rica. Mayo de 2013 a enero del 2014.

\begin{tabular}{lcccc}
\hline $\begin{array}{l}\text { Etapa de disección } \\
\text { (hojas emitidas) }\end{array}$ & $\mathbf{n}$ & $\begin{array}{c}\text { Plantas en fase } \\
\text { de transición o } \\
\text { reproductiva (\%) }\end{array}$ & Altura (cm) de la inflorescencia \\
\cline { 5 - 5 } & Hartón alto & & Mínimo $^{\mathbf{1}}$ & Máximo $^{2}$ \\
\hline 20 & 14 & 10 & 0,2 & 0,6 \\
22 & 14 & 80 & 2,0 & 9,5 \\
24 & 13 & 100 & 2,3 & 123 \\
26 & 14 & 100 & 21 & 283 \\
28 & 11 & 100 & 12 & 258 \\
& Hartón enano & & & 1,1 \\
20 & 10 & 60 & 0,2 & 42 \\
22 & 12 & 66 & 2,2 & 176 \\
24 & 16 & 100 & 8,0 & 150 \\
\hline
\end{tabular}

${ }^{1} \mathrm{y}^{2}$ Valor menor y mayor del conjunto de plantas (n) disectadas.

emisión de la inflorescencia, valor que correspondería al $90 \%$ de las plantas del cultivar alto y al $40 \%$ de las del cultivar enano.

Este proceso de transición continuó, en un porcentaje importante de plantas, en la hoja verdadera 22 y en muchas de ellas fue posible apreciar como el meristemo floral había iniciado su recorrido a través del pseudotallo. Esta particularidad indicaría el rango de hojas de la planta en que ocurrió tanto la transición floral como el ascenso inicial de la inflorescencia. A partir de la hoja 22 la amplia variación de la altura del meristemo reproductivo dentro de la planta, sería el factor responsable de la magnitud en la dispersión de la floración, y por consiguiente, de la cosecha.

Aunque en este estudio la variación estimada del número de hojas donde ocurrió la transición del meristemo fue de tres hojas (hojas 20 a 22), otros autores en banano (Dumas, 1955; Ganry, 1973) y en plátano (Hernández et al., 2010), reportaron rangos de emisión foliar más amplios.

Estudio IV- Relación de los cambios morfológicos de la hoja con la diferenciación floral. El cambio morfológico paulatino de la base de los semilimbos, desde totalmente aguzados hasta totalmente redondeados (Figura 2), ocurrió siempre con el meristemo en la fase vegetativa. No se determinó relación alguna entre el cambio morfológico, cuya duración fue de dos a tres hojas verdaderas, y el número de hoja verdadera emitida en lo que se inicia el cambio morfológico (Figura 3A). Este proceso inició en un 3,0\% de las plantas en la hoja verdadera 4, mismo que se mantuvo hasta la emisión de la hoja verdadera 14, en un 9,3\% de las plantas (Figura 3B).

De acuerdo con Belalcázar et al. (1991) la expresión visual de la diferenciación floral no solo guarda relación con un determinado porcentaje de hojas emitidas, sino también, con los cambios morfológicos que registran los dos semilimbos de la hoja en su parte basal. Sin embargo, en contraste con lo señalado por dicho autor, no fue posible encontrar en este estudio una relación entre la fase de diferenciación floral con el cambio morfológico de la parte basal de los semilimbos o la emisión foliar. Este aspecto concuerda con lo expuesto por Hernández et al. (2010) quienes señalan que la planta no manifiesta expresión visual externa alguna con respecto a la transición floral. 


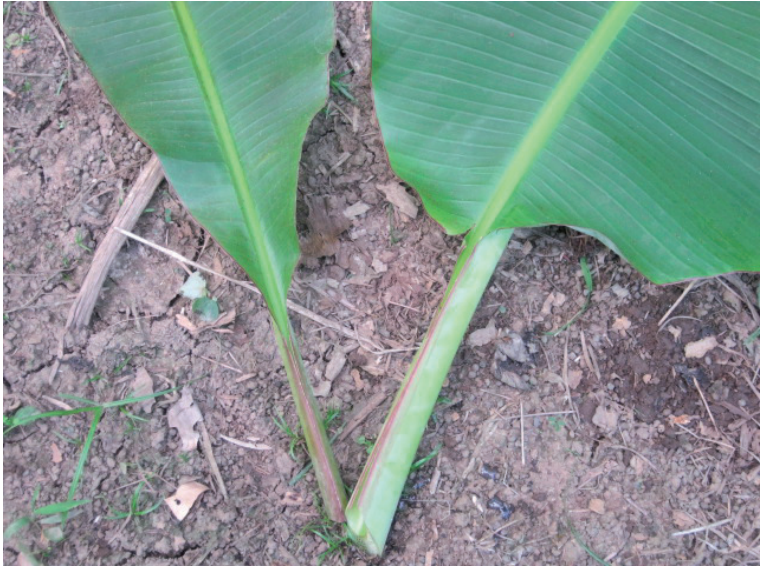

Figura 2. Variación morfológica de la base de los semilimbos (lóbulos) desde la última hoja emitida con ambos lóbulos aguzados hasta la primera hoja emitida con ambos lóbulos redondeados. Estudio IV. Matina, Limón, Costa Rica. 2012-2013.
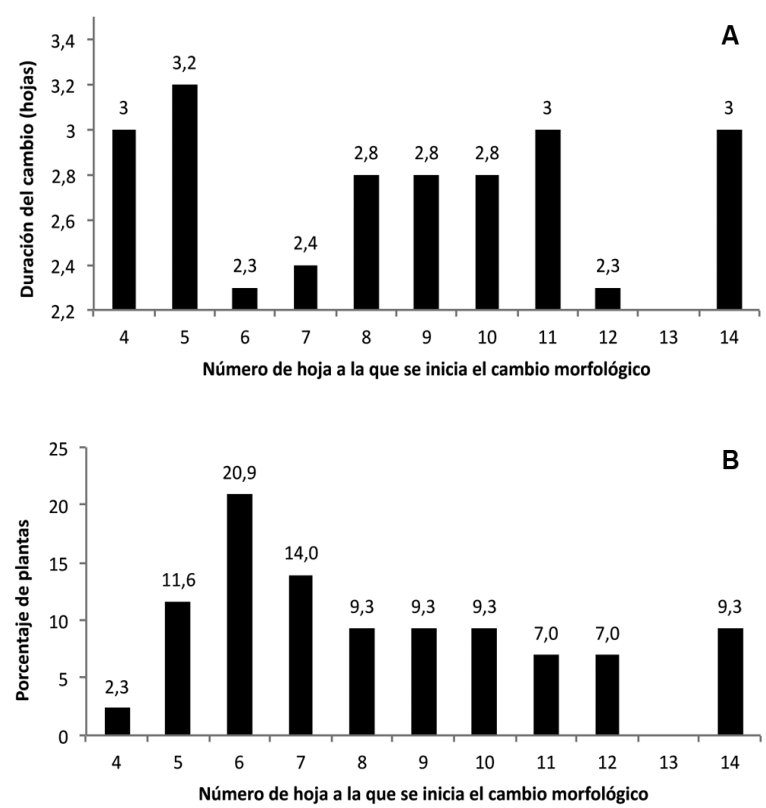

Figura 3. Cambio morfológico de los semilimbos de hojas de plátano: A- Duración (días) del cambio morfológico de los semilimbos de la hoja y el número de hoja en el que éste inicia: B- Porcentaje de plantas de acuerdo con el número de hoja a la que se inicia el cambio morfológico de los semilimbos. Estudio IV. Matina, Limón, Costa Rica. 2012-2013.

\section{LITERATURA CITADA}

Alexandrowicz, L. 1955. Étude du développement de l'inflorescense du bananier Nain. Annales. I.F.A.C. 9. Paris, France.

Aristizábal, M., y C. Jaramillo. 2010. Identificación y descripción de las etapas de crecimiento del plátano Dominico Hartón (Musa AAB). Agro. 18(1):29-40.

Belalcázar, S., y J. Valencia. 1998. Comportamiento de variedades de plátano. En: M. Giraldo et al., editores, Seminario Internacional sobre Producción de Plátano. Corpoica, Armenia, Colombia. p. 21-25.

Belalcázar, S., J. Valencia, y J. Lozada. 1991. La planta y el fruto. En: S. Belalcázar et al., editores, El cultivo del plátano en el trópico. Manual de Asistencia Técnica $\mathrm{N}^{0}$ 50. Impresora Feriva Ltda., Instituto Agropecuario Colombiano, Centro de investigaciones para el Desarrollo, Comité Departamental de Cafeteros del Quindío, Red Internacional para el Mejoramiento del Banano y el Plátano. Armenia, Colombia. p. 41-89.

Dumas, J. 1955. Contribution à l'étude du développent du bananier Nain. Fruits. 10:301-326.

Ganry, J. 1973. Étude du développement du système foliare $\mathrm{du}$ bananier en fonction de la temperature. Fruits. 28:499-516.

Hernández, Y., G. Giménez, y M. Gómez. 2010. Flowering Locus $T$ y Constan en Musa: nuevos genes que participan en la transición floral en plátano Musa AAB cv. Hartón Enano. Rev. Fac. Agron. (LUZ) 27:524-554.

Instituto Meteorológico Nacional. 2008. Cambio climático. Clima, su variabilidad y cambio climático en Costa Rica. Ministerio del Ambiente Energía y Telecomunicaciones (MINAET), Instituto Meteorológico Nacional (IMN), Programa de las Naciones Unidas para el Desarrollo (PNUD), Comité Regional de Recursos Hidráulicos (CRRH). San José, Costa Rica.

López, A., y P. Solís. 1991. Contenidos e interacciones de los nutrimentos en tres zonas bananeras de Costa Rica. CORBANA 15:25-32.

SAS Institute. 2004. SAS/STAT 9.1. User's guide. Version 9.1. ed. SAS Institute Inc., Cary, N.C., USA.

Serrano, E., R. Segura, R. Ortega, y J. Sandoval. 2008. Modelo de restitución del potasio removido en la fruta fresca exportada en una plantación de banano de alta productividad. En: J. Sandoval, editor, Informe Anual 2007. Corporación Bananera Nacional. Guápiles, C.R. 215 p. 
Stover, H. 1979. Pseudostem growth, leaf production and flower initiation in the Grand Nain Banana. Bulletin No 8. Tropical Agriculture Research Services, La Lima, Honduras.

Summerville, W. 1944. Studies on nutrition as qualified by development in Musa cavendishii Lambert. Queensland J. Agri. Sci. 1:128.

Turner, D., J. Fortescue, y S. Thomas. 2007. Environmental physiology of the bananas. Braz. J. Plant Pathol. 9:463-484.

Valle, H., y M. González. 2009. Determinación de la diferenciación floral en plantas de banano (Musa AAA) de cuarta generación en los cvs. Gran Naine, Valery y Williams. En: J. Sandoval, editor, III Congreso Científico Técnico Bananero Nacional. Corporación Bananera Nacional, Guápiles, Costa Rica. p. 51.

Valle, A., A. Segura, J. Sandoval W. Rodríguez, y M. González. 2007. Diferenciación floral en los cultivares de banano Grande Naine, Valery y Williams en plantas in vitro. En: J. Sandoval, editor, II Congreso Científico Técnico Bananero Nacional. Dirección de Investigaciones, Corporación Bananera Nacional, Guápiles, Costa Rica. p. 5.

Vargas, A. 1994. Validación de tecnología de producción para alto rendimiento en el cultivo del plátano Curraré o Falso Cuerno (Musa AAB) en el Atlántico de Costa Rica (primera cosecha). CORBANA 19:17-24.

Vargas, A. 1995. Validación de tecnología de producción para alto rendimiento en el cultivo del plátano Curraré o Falso Cuerno (Musa AAB) en el Atlántico de Costa Rica (segunda cosecha). CORBANA 20:29-31.

Vargas, A., P. Acuña, y F. Blanco. 2005. Caracterización morfológica y productiva de nueve cultivares de plátano Musa AAB Falso Cuerno. CORBANA 31:113.

Vargas, A., y F. Blanco. 2004. Metodologías para estimar la intensidad de desmane en racimos de banano (Musa AAA, cv. Valery). CORBANA 30:107-119.

Vargas, A., y M. Guzmán. 2001. Comparación de dos estrategias de combate químico de la Sigatoka negra (Mycosphaerella fijiensis Morelet) en plátanos del tipo Falso Cuerno (Musa AAB). CORBANA 27:65-78. 